\title{
Ранно откриване на нарушения на слуха при новородени
}

\author{
Ив. Ценев, М. Милков \\ Клиника по УНГ-болести - София \\ Специализирана УНГ-клиника "Св. Петка" - Варна
}

\begin{abstract}
The universal screening of hearing of newborns (USHN) is widespread in Europe and around the world, but at the moment still the results are not very well reported and documented. The initiation of such screening in Bulgaria is forthcoming.

The introduction of USHN is due to the personal contribution of pediatricians and audiologists. Simultancously with the device equipment and staff training of great importance is the tracking of children with hearing test abnormalities.

The consensus among the European audiologists says that the hearing disturbance must be diagnosed during the first 3 months after birth and between the $3 \mathrm{rd}$ and the 6 th month a hearing device must be found. For this purpose the screening- level 1 and 2 must be held in the newborn wards.
\end{abstract}

\section{Резюме}

Универсалният скрининг на слуха на новородените (УССН) намира все по-широко разпространение в Европа и света, като до момента не може да се говори за добро отчитане на резултатите. За България въвеждането на такъв скрининг предстои. Въвеждането на УССН се дължи преди всичко на личния принос на отделни педиатри и аудиолози. Успоредно с оборудването с апарати и обучението на отговарящия за изследването на новородените персонал, от съществено важно значение е и т. нар. „tracking“, това означава последващо наблюдение на децата с промени в теста.

Това става с помощта на повишаващо се административно участие и работа и подлежи на определени правила за защита на данните. Сред аудиолозите в Европа съществува консснсус за това, че в рамките на пьрвите 3 месеца на детето трябва да бъде поставена диагноза „намаление на слуха“, а между 3-ти и 6-ти месец да се започне с тьрсене и доставка на слухов апарат. За целта още в родилните отделения трябва да се провсждат скрининг степените 1 (обичайно измерване по TEOAE) и 2 (AABR - тестуване). Проследяването на стеген 1 при децата с отклонения се състои в повторение на TЕОАЕ и AABR измерването при лекари специалисти. Проследяването на степен 2 обхваща потвърждаваща диагностика в педиатрично - аудиологичен център. Това винаги включва и BERA диагностика при спонтанен сън или седиране. Осигуряването на слухов апарат по правило трябва да стане при специализиран за деца (псдиатьр) акустик.

\section{Въведение}

Вродените нарушения на слуха са най-честата вродена сензорна малформация. При здраво родени бебета (well - babies) те възлизат на 2 на 1000, при деца с риск (напр. фамилна глухота, пре-перипостпартални инфекции, преждевременно родени с тегло под 1500 грама, изискваща трансфузия хипербилирубинемия, краниофациални дисморфии и т.н.) дори достига до $50[1,2]$. Докато процесът на „узряване“ на пътя на зрението завършва в кърмаческа възраст до 6-тия месец [3], то този процес при пътя на слуха започва от раждането и е до края на първите 4 години. Тъй като за невроналните мрежи и връзки за „узряване“ на слуховия път се изисква постоянно и достатъчна информация, от съществено значение е правилното функциониране на средното и вътрешното ухо. Ако има подлежащо намаление на слуха, това съзряване на пътя на слуха не може да се осъществи или е непълно и недостатъчно и развитието на слуха претьрпява необратими увреждания (последствия) (промени). Без функциониране на слуховия апарат е невъзможно нормално говоримо, интелектуално и социално развитие [4-8]. Нормалното интелектуално и училищно развитие при късно диагностицирано намаление на слуха е възпрепятствано и след навършване на 4 години, дори и при оптимално въздействие теоретичният умствен потенциал на детето не може да бъде развит $[8,9]$. По тази причина всички медици по света се стремят да изследват слуха на новородените няколко дни след раждането, за да могат в случай на доказване на намален слух да започнат да действат за доставка на слухов апарат в първите 3 до 6 месеца $[10,11]$. Не всички автори могат да докажат зависимостта между ранното 
откриване на намалението на слуха за вътрешното ухо и последващото осигуряване на слухов апарат с обусловеното от това позитивно въздействие върху говорното развитие [12]. Институтът по качество и икономика в здравеопазването (ИКИЗ) казва, че има „насоки, тенденции, но не и доказателства“ за това, че „при универсалния скрининг на слуха на новородените диагностицираните деца с нарушения в слуха имат предимства при развитието на говора“" [13]. Въпреки това е налице причинно-следствена връзка, която признават многобройни експерти от цял свят, което е установено от УССН в много континенти и много страни. В Германия се стремят към единен скрининг [14-22], но въпреки това за момента само в отделни региони и бундес-държави има УССН (изобр. 1) [23-27]. Много други страни, сред които и Африка $[28,29]$, Близкият Изток [31, 32] и Далечният Изток [32-35] опитват да въведат такъв универсален цялостен покриващ плоскостта скрининг на слуха на новородени УССН. Сред тях са страните под прага (от третия свят) и развиващите се страни като $[32,35$, 36] Малайзия [35], Тайланд [32], Мексико [37] и Индия [38]. В Европа съществуват многобройни стремежи да се въведат изследвания за скрининг на слуха при новородени. Публикации по тази тема има не само от Северна [39], Средна [40-42] и Южна Европа [2, 42-44], а също и от Сърбия [45], Хърватия [46], Албания [47] и Грузия [48]. Понастоящем покриващ плоскостта УССН се осъществява в следните страни и континенти: Европа (Белгия, Холандия, Португалия, Австрия [49, 50], Англия, Полша); в Австралия, Северна Америка (САЩ) и Канада $[51,52]$; Централна Америка (Куба); Азия - напр. Малайзия [35]. Някои страни, напр. Китай [53], предприемат дори и идеята да въведат такава скрининг програма.

В Китай има програми, които свързват скрининг на зрение и слух [53]. В Австрия по финансови причини (спестяване на време при измерванията) се провежда един скрининг на ухото. Там се казва, че недоказаното и нелекувано едностранно намаление на слуха може да повлияе неблагоприятно на социалното и когнитивно развитие на деца с увреден слух. Като при това тези деца имат поголям риск от проблеми в училище, отколкото нормално чуващите деца $[54,55]$.

Програми за въвеждане на изследванията за скрининг на слуха при новородени (УССН) действат от няколко години по света [32, 56-60]. Една статия в New England Journal of Medicine озаглави това още през 2006 г. като „,тихата революция“ („Newborn hearing screening - a silent revolution") [61]. Преди повече от 20 години в Германия Matschke и Peath, основавайки се на изследването на слуха при децата, са предложили да се провеждат съответни повсеместни изследвания [62]. Като причина за нарушенията на слуха при новородени в над 50\% от случаите се отбелязват вродени нарушения на слуха - генетични, които в преобладаващата част от случаите се свързват с GJB2 или GJB6 - мутации [51, 63-68]. Различават се синдромални и несиндромални нарушения на слуха [69].

\section{Доказателства (данни) за намалението на слуха при деца}

Причината, поради която сыществуват усилията, за да се въведе УССН, се корени най-вече в честотата на вродените увреди на слуха. Както вече беше описано по-горе, те са най-честите вродени сетивни нарушения (малформации) и са по-чести от всички заболявания взети заедно, за които досега се провеждат крьвни скрининг тестове при новородени. Едно на 500 до 1000 новородени деца има най-малко средно по степен сензори - невронално намаление на слуха $[4,70,71]$. Така например данните от едно изследване на Finck - Kramer и сътрудници от 1998 г. показват, че средната възраст на първоначално диагностициране на граничещите с глухота намаления на слуха за вътрешното ухо (НСВУ) е 19 месеца, при високостепенно НСВУ е 2 години, средностепенно НСВУ - 4 години и при нискостепенно - 6 години [74]. В действителност средното подозрение за това се е появило няколко месеца по-рано - времето за осигуряване на слухов апарат е било още по-късно [10. 74]. При това може да се докаже, че възрастта на първоначално диагностициране може да се премести съществено по-напред с помощта на УССН $[54,75]$. Средната възраст на децата, при която е поставена първоначална диагноза намаление на слуха през 2006 г. според Хесингския модул, е само 3,1 месеца [54].

\section{Технически възможности за диагноза}

За тестуване на слуха при новородени от години съществуват голям брой методи [76-78], които позволяват да се използват обучени за кратко време хора, без специализация в областта и като цяло по-ниско квалифицирани (по-ниско платени) изследващи и за секунди или минути да се изследва способността за чуване на спящо или почиващо дете. TEOAE и AABR - изследването са въведени с такава цел при УСCH. В тази връзка DPOAEтестванията не се препорьчват, тъй като полученият праг на чуване при този метод е 40-45 dB и така ниско- до средностепенните нарушения на слуха за вътрешното ухо остават неоткрити [79]. Трябва 
да се откажем от представянето на техническите основи на този метод (посочена е съответната литература). Направеното TEOAE-тестване доказва слух, по-добър от $30 \mathrm{~dB}$, а AABR - измерването - слух, по-добър от $35 \mathrm{~dB}$ [77, 79].

Аудиторните (слухови) невропатии могат да бъдат доказани чрез AABR - скрининг $[80,81]$. Едно изследване на Foerst и сътр. показва, че това заболяване при високостепенно намален слух у децата е с $8,44 \%$ по-често, отколкото се е смятало досега [80]. По-нискостепенното намаление на слуха за вътрешното ухо поради споменатите вече причини не може да бъде сигурно изключено чрез неспецифичната находка от скрининг теста за слуха. Затова по правило по-нискостепенното намаление на слуха за вътрешното ухо се диагностицира покъсно от високостепенното. Възрастта на поставяне за първи път на диагнозата нискостепенно намаление на слуха за вътрешното ухо в Германия е приблизително чак към 6 години [74].

Публикуваните в NEJM работи на Paradise и сътр. показват, че нелекуваните изливи в кухината на средното ухо, които могат да доведат до нискостепенно намаление на слуха, не водят до сериозно влошаване на развитието на говора $[82,83]$.

Това обяснява късното поставяне на диагнозата на нискостепенните намаления на слуха. Въпреки това трябва да се има предвид, че намалението на слуха с 30 dB има същия ефект както и използването на запушалки за шум на ушите (антифони). Праг на чуване от $30 \mathrm{~dB}$ е еквивалентен на увеличение на разстоянието при разговорна реч между човека и личността, на която говори на 30 м [84].

\section{Последващи разходи при намаление на слуха при деца}

Консенсусът на DGPP, който бе приет на годишното заседание през 2007 г. в Инсбрук, гласи, че дори един среден праг на чуване между 20 и $25 \mathrm{~dB}$ може да бъде индикация за опит да се нагласи по-точно слуховият апарат [85]. При средно и високостепенно намаление на слуха не може да се осъществи нормално говоримо и интелектуално развитие на децата [86]. При високостепенно намаление на слуха на децата се взема решение според времето, в което е доказано, и лечението, което ще се предприеме, да поемат пьт още от ранните си години за образование и професионална квалификация в обшество, в което могат да съществуват. Следователно ранната диагностика на намаления слух има освен влияние върху индивида и върху икономиката като цяло. От една страна, чрез подобрите възможности за развитие на индивида, от друга страна - чрез разходите, които се получават за медицинско обслужване и различни форми на обучение (обичайни или специални училища) $[54,87,88]$. Разходите за едно тежко чуващо дете, при което чрез УССН е доказано високостепенно намаление на слуха, възлизат на $13438 €$, а при скрининг на риска - на само $7241 €$ и без систематичен скрининг - на $4760 €$ [54].

\section{Начин на процедиране при въвеждане на УССН}

Когато днес говорим за въвеждане на качествен гарантиран УССН, покриващ плоскостта, ние имаме предвид следното:

Най-малко $95 \%$ от новородените трябва да бъдат тествани за слуха в първите дни от живота си; максимално $4 \%$ от тях са с отклонения в теста; важно е да се обхванат всички със средна и висока степен на намаление на слуха; трябва да бъде осигурен tracking на пациентите; своевременен follow up (проследяване) в специализирани педиатрични аудиологични центрове е възможно и така се постига външно гарантиране на качеството [4, $70,71,96]$.

Най-напред създаване на теоретични основи.

- В страната трябва да има списьк (инвентаризация) кои клиники предлагат тестване на слуха на новородени и кои (все още) не. Трябва да се изясни дали по възможност може да има спонсорство.

- Трябва да бъде издействан политически мандат, като паралелно се започне с планирането на УССН.

- Трябва да се постигне мултидисциплинарен консенсус. Това означава всички, участващи в УССН групи от различни професии (акушери, детски УНГ лекари, педиатри - аудиолози, специалисти по грижите, тьрговски представители и др.), трябва да се съберат на една маса.

- Трябва да бъде разработена концепция за протичането на УССН. Тя може да съдържа IT и съхранение на данните. Трябва да се формулира изследователски проект с етична част и трябва да е сигурно, че използваните апарати за скрининг, обучението на скриниращите ще осигурят безгрешно врыщане обратно на информацията за резултатите към скринингцентъра. Възможните възникнали грешки на скриниращия трябва по всяко време да могат да се коригират.

Фазата на практическото провеждане на УССН изисква следните точки (пунктове): 
- Осигуряване на информация и обучение на всички активно участващи в скрининга.

- Обосноваване на общополезен съюз за подпомагане с цел набиране на средства, търсене на спонсори.

- Започване на обществено разгласяване (преса, телевизия).

- Оборудване със скрининг апарати на всички родилни и детски клиники.

- Осигуряване на покриващ плоскостта последващ скрининг и проследяване чрез обучение и сертифициране на избрани направления (практики и клиники).

- Начини на оповестяване и съхраняване на данните от съответна служба за съхраняването им към бундес - страната. Едва след това могат да бъдат съобщени данните.

- Организиране на периодични обучения на всички участващи - централно и по места.

Накрая към другите се добавя и изискването за научна оценка на резултатите, тяхното публикуване в специализиран кръг, както и постепенните опити за оптимизиране на процеса [93].

При въвеждането на УССН ръководните хора трябва да вземат решение дали трябва да се провежда едно- или двустепенен скрининг. При чистия рисков скрининг на деца с по-горе описаните рискови фактори се стига до там, че в $42 \%$ високостепенно намаление на слуха не може да бъде открито и затова се смята за недостатък [97].

Двустепенният скрининг (напр. инициативата $\mathrm{ABCD}$ в областта Nordrhein в Германия) се провежда при деца без риск „well babies“ - пьрво TEOAE измервания. Децата без отклонения (критерий за преминаване) не трябва да бъдат изследвани впоследствие, докато тези с изменения трябва да бъдат подложени на AABR - измерване [53, 54, 98-100]. Рисковите деца се тестуват с AABR и след това се повтаря това измерване, ако при първия метод има "refer" .

\section{Рискови фактори за нарушения на слуха при децата}

Като рискови фактори се смятат следните белези: нарушения на слуха във фамилията, вирусно обусловени интраутеринни инфекции в първите 5 месеца от бременността (напр. шарка или цитомегаловирус) и лечение с ототоксични или тератоксични медикаменти в първите пет месеца. Недостигът на кислород при раждането, тегло при раждането по-малко от 1500 г, АПГАР под 4, изискваща трансфузия хипербилирубинемия, малформации в областта на главата, краниофациал- ни дисморфии, синдроми, хромозомни оберации, преждевременно раждане преди 32 гестационна седмица, интраутеринна регардация, близкородственост на родителите, зависимост на майката, перинатална асфиксия, обдишване повече от 10 дни, тежки респираторни нарушения в адаптацията, черепно-мозъчни травми или др. [100, 101].

\section{Модельт Хеснер}

При модела Хеснер, който е разработен под рьководството на Катрин Нойман и Петер Бьотчер, е възможно чрез комбиниран метод (двустепенен модел) да се постигне повишаване на специфичността от $95 \%$ на $97 \%[54,55]$. Комбинирането на двата метода има и следните предимства: предпочитането първо на ТЕОАЕ-измерването, което в сравнение с AABR трае по-кратко време. Също и разходите за изразходвани материали са по-малко при ТЕОАЕ-измерването [103, 104]. А преимущество на AABR-измерването е неговата по-голяма специфичност и сензитивност $[76,19]$. Двустепенният модел се смята като по-изгоден икономически $[54,106]$. Но в сравнение с чистия AABR-скрининг има недостатък, че аудиторните невропатии могат да останат неразпознати [80]. При „,well babies“ само в редки случаи може да има и при нормален TEOAE-тест теоретично високостепенно сензоневронално намаление на слуха, обусловено от аудиоторнална невропатия. Относителният дял на аудиоторните невропатии при високостепенно намаление на слуха възлиза на над повече от $10 \%$ [80]. Ако и при втората стъпка от изследването на скрининга на слуха има отклонения, по правило трябва да бъде проведена още в родилния дом, трябва в рамките на 2 седмици да се проведе по-нататъшно педиатрично-аудиологично изследване в специализиран център [4]. Тук при follow up степени 1 и 2 влизат в съображение поред с биокуларно микроскопските изследвания и субективни тестове с помощта на аудиометрии в свободно поле, мултичестотни тимпанометрични измервания, диагностични OAE-тествания и BERA метод при седация [77]. Освен Click-BERA изследванията, които обхващат областта между 1500 и $4000 \mathrm{~Hz}$, могат да бъдат проведени и how Chiop BERA (250-850 Hz) или Notched Noise BERA, които имат възможност селективно да изследват областите на 500, 1000, 2000 и $4000 \mathrm{~Hz}$. При кърмачета, при които бива доказано намаление на слуха за вътрешното ухо, трябва още между 3-тия и 6-тия месец да се постави слухов апарат от педиатьр-акустик. Процесьт на напасване е динамичен. Педиатьрьт-акустик 
или аудиолог съпровождат детето с години [15]. Половин година след първото напасване на слуховия апарат трябва да се проведе контролно BERA - изследване, т.е. в идеалния случай на 9 месеца, тъй като има случаи, при които със съзряването на пътя на спуха се подобрява и прагът на чуване [107]. Във възрастта 9 месеца се потвърждава диагнозата чрез контролното BERA-изследване, тъй като първата BERA на 3 месеца е била своеобразна „работна хипотеза“. Ако намалението на слуха на засегнатото дете е твърде изразено, така че трябва да се лекува със слухов апарат, тогава съществува възможност още преди навършване на 1 година да се постави кохлеарен имплантант (CI) [108]. За съпътстващата рехабилитация са на разположение специални отделения, най-често в университетските центрове. От изследванията на Хесен стана ясно, че 56\% от прелингвално глухите деца, които са снабдени с кохлеарен имплантант след 3-тия си рожден ден, не могат да посещават обикновени детски градини и училища, докато при тези с имплантант, поставен преди навършване на 3 години, дельт е 24\% [54]. Kohski и сътр. са изследвали при едно проучване във Франкфурт по кое време се прави скринингът за слуха на децата, непосредствено след раждането или 2 месеца по-късно при преглед от педиатър. То показва, че $95,72 \%$ от децата са тествани в родилния дом и 64,18\% при преглед на 2 месечна възраст.

По-нататьшните изследвания могат да докажат, че при програмите за скрининг на слуха, ако няма качествен follow up (проследяване), повече от $50 \%$ от децата не се подлагат на по-нататьшно изясняване на диагнозата, дори и при първично положителен тест от скрининга на слуха [110]. Тук се говори за host follow up - квота $\{54,110]$. Тя може да бъде редуцирана на 7,8\% според модела на Хесен чрез централно обхващане на децата и насочване на родителите към съответните центрове за допълнителни изследвания. Достигането на такава ниска квота е свързано с интензивна работа в скрининг - центровете. Така например Bottcher съобщава, че през 2006 г. в Хесен при УССН са били обхванати общо 34133 деца. От тях при 2220 е бил необходим tracking. От скрининг - централата са били проведени 4340 телефонни разговора (956 от които са по сигнал от родилни клиники) и са били изпратени 2890 писма. При 66 деца е било потвърдено намаление на слуха [55]. Това показва, че успешният tracking е с радостно ниска "lost-to-follow up" квота 7,8\%, свързан с интензивна работа във времето и обществото.

В родилното отделение се прави най-напред задължително изясняване на младите родители в устна и писмена форма. Предимството на скрининг-ID пред индивидуалното въвеждане на данните е автоматичната извадка на персонални данни и избягването на грешки или повторно нанасяне. Скрининг-ID могат да се използват и напр. за други скрининг-форми като например скрининг за обмяната на веществата. След съгласието на родителите за скрининг и провеждането на теста резултатите се нанасят и документират в жълта тетрадка за прегледи и впоследствие заедно със скрининг-ID се заключват и предават в скринингцентьра. Времето за провеждане на скрининга на здрави и доносени (зрели) новородени е преди изписване от родилното отделение, а при по-рано родени - скоро преди изписването, по възможност преди да се достигне терминът на раждане [70].

Ако резултатът от скрининга е с отклонения, се преминава към втора степен на скриниране. Новото тестване на слуха трябва да стане по възможност в рамките на 4 седмици при фониатър/ педиатър-аудиолог, респ. педиатрично-аудиологично квалифицирани УНГ лекар или педиатър (2-ри скрининг). Също и децата, които са родени амбулаторно (в дома), трябва да подлежат на пьрвия си скрининг на слуха при тези лекари. Това важи и за случаите, когато при първото тестване е изследвано само едното ухо.

Качеството на апаратите за скрининг се обезпечава от производителя, докато персоналът е отговорен за поддръжка и калибриране [112].

Педиатрично-аудиологичното потвърждение на диагнозата (follow up степени 1 и 2) става тогава, когато втората степен на скрининга също е с отклонения.

Ако детето има резултат с отклонения, то се изследва в същото заведение преди изписването му още един път (повторно). Ако и този резултат е същият, се въвежда първата стъпка от двустепенния follow up. При follow up степен 1 първо се насрочва час за преглед за специален скрининг и след това се продължава с повторение на TEOAЕ и AABR-измерване. След това бинокулярно микроскопски се снима находка от ухото. При необходимост могат да влязат в съображение и други методи за изследване като тимпанометрия или изследване на свободните полета на масата за деца на Майнд.

При follow up - степен 2 се провежда по-нататъшна педиатрично-аудиологична диагностика в специализиран центьр. Тук с помощта на BERAизследване се осъществява потвърждаване на диагнозата, която вече се поставя окончателно за наличие или липса на нарушение на слуха, което да изисква или не доставка на слухов апарат. Тази 
диагностика трябва да приключи в рамките на първите 3 месеца и да бъде последвана от нагласяне на слуховия апарат в първите 6 месеца $[70,71,112]$. Първите скрининг-прегледи, които започват още в родилния дом, се провеждат от сътрудници, които са преминали успешно предварително специален курс на обучение. Такива курсове се провеждат от сътрудници на педиатрично-аудиологичните центрове (2-ра степен на follow up) или ръководни (методични) места за провеждане на скрининг. От тези данни след това се обобщават отново „впечатляващи“, респ. „невпечатляващи“ находки, общите данни, фалшиво-позитивни и фалшиво-негативни находки, както и критерии за качество на скрининга. От тях могат да се изведат важни по-ната-

\section{Литература:}

1. Henke KD, Huber M. Neonatales Horscreemng - gesundheitspolitische Konsequenzen. Gesundheitswesen 1999; 61: 86-92

2. De Capua B, Costantini D, Martufi C, latini G, Gentile M, De Felice C. Universal neonatal hearing screening: The Siena (Italy) experience on 19700 newboms. Early human development 2007; 83: $601-606$

3. Lelinhardt E. Zur sensiblen Phase der Horbahnreifung - Parallelenzum Auge Laryngo Rhino Otol 2004; 83: 766-767

4. Interdiszipliniire Konsensus-Konferenz fur das NeugeborenenHorscreening. Universelles Horscreening bei Neugeborenen. Empfehlungen zu Organisation und Durchfilhrung des universellen Neugeborenen-Sere en ings auf angeborene Horstorungen in Deutschland. HNO 2004: 52: 1020-1027

5. Chiong C Osrrea E jr. Reyes Л, Ltanes EC. Uy ME. Chan A. Correlation of hearing screening with developmental outcomes in infants over a 2-year period. Acta oto-laryngologica 2007; 127; 384-388

6. Fitzpatiick E, Duricux-Smith A, Eiiks-Brophy A, Olds J, Gaines R. The impact of newborn hearing screening on communication develop- - ment. Journal of medical screening 2007; 14: 123-131

7. Kennedy CK McCann DC, Campbell MJ, Law CM. Muilee M, Petrou 5, Watkin P, Worsfold S, Yuen HM, Stevenson J. Language ability after early detection of permanent childhood hearing impairment. The New England journal of medicine 2006; 354: 2131 -2141

8. Lohie E. Holm M. tehnharcit E. Preconditions of language develop $\neg$ ment in deaf children. International journal of otorhinolaryngology 1999; 47 : 171-175

9. Kennedy CR, McCann DC, Campbell MJ, Law CM, Mullee M. Petrou S, Watkin P, Worsfold S, Yuen HM, Stevenson ]. Language ability after early detection of permanent childhood hearing impairment. The New England journal of medicine 2006: 354: 2131 -2141

10. Scliade G, Fleischers, Breitfuss A, Hess M. Friihversorgung mit Horgeraten in den ersten Lebensmonaten. HNO aktuell 2008; im Druck

11. Fleischer S Hess M. Besonderheiten der Horgerateversorgung im Sauglings-. Kleinkind- und Vorschulalter. HNO 2002; 50: 501 - 510

12. Fitzpatrick E, Graham ID, Durieux-Smith A, Angus D, Cayte D. Parents' perspectives on the impact of the early diagnosis of childhood hearing loss. International journal of audiology 2007; 46: 97- 106

13. Institut Д1г Qualitai und Winschaftiicltkeit im Gesundheitswesen. Absdilusshericht Friiherkennungsuntersuchung von Horstorungen bei Neugeborenen. IQWiG 2007 (1. Auflage) pdf www: 1 - 300

14. Keijmann A. Zum Konsensus-Papier zum universellen NeugeborenenHorscreening in Deutschland. Laryngo-Rhino-Otol 200.1; R4:799-800

15. Lohle E. Moddlefurein universelles Neugeborenen-Horscreening in der Diskussion. HNO 2004; 52: 959-962

16. Pohiatidt F. Universelles Horscreening bei Neugeborenen. Empfehlungen zu Organisation und Durchftihrung des universellen Neugeborenen-Screenning auf angeborene Horstorungen in Deutscliland. K i nd e rkranke rise h wester 2005; 24: 239-244

17. Ptoc M. Grundlagen fur das Neugeborenen-Horscreenins (Standard of Care), Stellungnahme der Interdisdplinaren Konsensuskonferenz тъшни епидемиологични и икономически изводи. Анализът ,цена - полза“ и икономическата оценка на УССН трябва да се изведат от тези данни [112].

\section{Заключение}

Но само с помощта на политиците в Европа и България може да се осигури УССН да бъде действително покриващ плоскостта и продължителен. Остава да се надяваме, че персоналният ангажимент на много педиатри-аудиолози, УНГ-лекари, така ще ги движи, че България ще се причисли чрез оптимизиране на УССН към пионерите в света по успешно тестване на слуха на новородени и кърмачета.

Neugeborenen-Hiirscreening (IKKNHS). HNO 2003; 51: 876-879

18. Ptok M. Cirundlagen fur das Neugeborenen-Horscreening (Standard of Care), Stellungnahme der Interdisziplinaren Konsensuskonferenz Neugeborenen-Hurscreeniiig (IKKNHS). Zeitschrift fur Geburtshilfe und Neonatologie 2003; 207: 194- 196

19. Schonweiler R. Leserbrief zum Eeitrag von M. Cross - Universelles Horscreening bei Neugeborenen - Empfehlungen für Organisation und Durchführung des universellen Neugeborenen hois freenings auf angeborene Horstorungen in Deurschland Laryngo-Rhino-Otol 2005; 84:801-808

20. Schnetl-tnderst P, Kunze S, Hessel F, Grill E, Siebert U. Nickisch A. von Voss H, Wasem J. Horscreening für Neugeborene - Update 2006. CMS Health Technical Assess 2006; 2; Doc 20

21. Welzl-Muller K. Neugeboreneii-Horscreening: Sicbtest nach Horsturungen bei Neugeborenen. HNO 1993:46: 704-707

22. Wtibben J. Horstorungen bt'i Kindern: Frilberkennung zweigleisig verbessern Quellc. Deutsches Arzteblatt 1997; 94: 657

23. Shehata-Dieler WE, Dieler R, Wenzel C, Keim R. Singer D, von Deuster Ch. Das Wurzbuiger Horscreening-Programm bei Neugeborenen. Erfahrungen bei mehr als 4000 Sauglingen - Einfluss nichtpathologischer Faktoren auf die Messergebnisse. Laryngo Rhino Otol 2002; 81:204-210

24. Plinkert PK, Delb W. EDV-gestiitzter Aufbau eines interdisziplinaren landesweiten Horscreenings im Saarland. HNO 2001; 49: $88 \breve{\text { - }} 894$

25. Nennstiel-Ratzei U, Arem S, Kries R von, WMwr M. Strutzj. Modellprojekt Neugeborenen-Horscreening in der Oberpfalz: Hohe Prozessund Ergebnisqualitat durch interdisziplinares Konzept. HNO 2007; 55 : 128-134

26. Kchrt W, Geidel L, Wilkens Ш, Lohler J. Universelles NeugeborenenH6screening im Marienkrankenhaus Hamburg von September 1999 bis April 2002. Laryngo Rhino Oiol 2003: 82: 479-485

27. Schorn K. The Munich screening programme in neonates, British journal of audiology 1993; 27: 143-148

28. Swanepoel D. Ebrahim S, Joseph A, Friedland PL Newborn hearing screening in a South African private health care hospital. International journal of pediatric otorhinoUiryngology 2007; 71: 881-887

29. de Swanepoel W, Hugo R, Louw B. Infant hearing screening at immunization clinics in South Africa. International journal of pediatrit otorhinolaryngology 2006; 70: 1241-1249

30. Al-Kandari JM, Atsftuaib WB. Newborn hearing screening in Kuwait. Electromyography and clinical neurophysiology 2007; 47: $305-313$

31. Khandekar R, Khabori M, Jaffer Mohammed A, Gupta R. Neonatal screening for hearing impairment - The Oman experience. Interna-tional journal of pediatric otorhinolaryngology 2006: 70: 6S3 - 670

32. Srisuparp P. Gleebbur R, Ngerncham S, Chonpracha J, Singkampong J. High-risk neonatal hearing screening program using automated screening device performed by trained nursing personnel at Siriraj Hospital: yield and feasibility. Journal of the Medical Association of Thailand 2005: 88:176-182

33. Lam BC. Newborn hearing screening in Hong Kong. Hong Kong medical journal 2006: 12:212-218 
34. Kaga K, Shinjo Y, Yamasobu T, Ito K Akamatsu V, Uchiyama 1", Tokumitsu $И$. Development of hearing, speech and language in congenially deaf infants and children after cochlear implantation. Brain and development 2007; 39: 335-345

35. Abdullah A, Hazim MY, Almyzan A, Jamilah AC Roslin S, Ann MT Borhan I, Sam A, Saim i, Boo NY. Newborn hearing screening: experience in a Malaysian hospiral. Singapore medical journal 2006: 47: 60-64

36. de Swanepoel W, Louw B, Hugo R. A novel service delivery model for infant hearing screening in developing countries. International jour nal ofaudiology 2007; 46: 321-327

37. Yee-Aretlano HM, Leal-GarzaF, Pauii-Miiller K. Universal newborn he aring screening in Mexico: results of the first 2 years. International journal of pediatric otorhinolaryngology 2006; 70: 1863-1870

38. Rao S, Patricia PL, Core M, Dominic M. Universal hearing screening. Indian journal of pediatrics 2007; 74: 545-549

39. Nielsen Ш, Konrddsson K. Universel neonatal twarescreening. Dansk Selskab for Otolaryngologi, Hoved-og Halskirurgi 2006; 168:1237

40. ZehnderA, Probst R, VischerM, Under T. Erste Resultate des allgemeinen Neugeborenen-Horscreenings in der Schweiz. Schweizerische medizinische Wochenschrift 2000; 125: 71 -74

41. Leveque M, Schmidt P, Leroux B, Danvin JB, Langagne T, Labrousse M, Chays A. Universal newborn hearing screening: a 27-month experience in the French region of Champagne-Ardenne. Acta paediatrica 2007; 96: 1150-1154

42. Dorba A, Hatzopoulos S, Camurri L, Negossi L, Rossi M, Cosso D, Petruccelli J, Martini A. Neonatal newborn hearing screening: four years' experience at Ferrara University Hospital (CHEAP project): part 1. Acta otorhinolaryngologica Italica 2007; 27: 10-16

43. Calevo MG, Mezzano P, Zullino E, Padovani P, Scopesi F Serra G Neonatal hearing screening model: an Italian regional experience. The journal of maternal-fetal \& neonatal medicine 2007; 20: 441 -448

44. Calevo M, Mezzano P, Zullino E, Padovani P, Sena G. Ligurian experience on neonatal hearing screening: clinical and epidemiological aspects. Acta paediatrica 2007; 96: 1592-1599

45. Sente M, Aleksov-Hatvan G. Early detection of hearing impairment in children. Srpski arhiv za celokupno lekarstvo 2006; 134: 448-452

46. Prpic I, Mahulja-Stamenkovic V, Bilk I, Haller H. Hearing loss assessed by universal newborn hearing screening - The new approach. International journal of pediatric otorhinolaryngology 2007; 71: 1757-1761

47. Hatzopoulos S, Qirjazi B, Manini A. Neonatal hearing screening in Albania: results from an ongoing universal screening program. International journal of audiology 2007; 46: 176-182

48. Burdzgla 1, Pietsch M, Chkhartishvili B, Kevanishvili Z. The proper time for hearing screening in newborns. Georgian medical news 2007: 24-27

49. Weichbold V, Nekahm-Heis D, Welzl-Muller K. Ten-year outcome of newborn hearing screening in Austria. International journal of pediatric otorhinolaryngology 2006; 70: 235-240

50. Weichbold V, Nekahm-Heis D, Welzl-Muller K. Zehnjahre Neugeborenen-H6rscreening in Osterreich. Eine Evaluierung. Wiener klinische Wochenschrift 2005; 117: 641-646

51. Putcha GV, Bejjani BA, Bleoo S, Booker JK, Carey JC, Carson N, Das S, Dempsey MA, Gastier-Foster JM, Greinwald JH Jr, Hoffmann ML,Jeng LJ, Kenna MA, Khababa I, Lilley M, Mao R, Muralidharan K, Otani IM, Rehm HL, Schaefer F, Seltzer WK, Spector EB, Springer MA, Week KE, Wenstrup RJ, WithrowS, Wu BL, Zariwala MA, Schrijverl. A multicenter study of the frequency and distribution of GJB2 and GJB6 mutations in a large North American cohort. Genetics in medicine 2007; 9: 413-426

52. MacNeil JR, Liu CL, Stone S, Farrell J. Evaluating families' satisfaction with early hearing detection and intervention services in Massachusetts. American journal of audiology 2007; 16: 29-56

53. Nic WY, Wu HR, Qi YS, Lin Q, Zhang M, Hou Q, Gong LX, Li H, Li YH, Dong YR, Guo YL, Shi JN, Yin SY, Li PY, Zhang WH. Simultaneous screening program for newborms hearing and ocular diseases. Chinese journal of otorhinolaryngology head and neck surgery 2007 ; 42:115-120

54. Neumann K, Gross M, Bottcher P, Euler HA, Spormann-Lagodzinski M, Polzer M. Effectiveness and efficiency of a universal newborn hearing screening in Germany. Folia phoniatrica et logopaedica: official organ of the International Association of Logopedics and Phoniatrics (IALP) 2006; 58: 440-455
55. Bottcher P. Qualitatsgesichertes Neugeborenen-Horscreening: Luxus oder Notwendigkeit? Vortrag in Miinster beim Symposion "Horscreening in NRW", am 5.9.2007

56. Grill E, Hessel F, Siebert U, Schnell-Inderst P, Kunze S, Nickisch A, Wasemj. Comparing the clinical effectiveness of different new-born hearing screening strategies. A decision analysis. BMC public health 2005; 5: 12

57. Kiese-Himmel C, Kruse E. Horstorung im Kindesalter. Wer hat als Erster den Verdacht? Deskriptive Analysen. HNO 2005; 53: 810-814

58. Weichbold V, Nekahm-Heis D, Welzl-Muller K. Universal newborn hearing screening and postnatal hearing loss. Pediatrics 2006; 117:631636

59. Mukari SZ. Tan KY, Abdullah A. A pilot project on hospital-based universal newborn hearing screening: lessons learned. International journal of pediatric otorhinolaryngology 2006; 70: 843 - 851

60. Grill E, Uus K, Hessel F, Davies L, Taylor RS, Wasem J, Bamford J. Neonatal hearing screening: modelling cost and effectiveness of hospital and community-based screening. BMC health services research 2006; 6: 14

61. Morton CC, Nance WE. Newborn hearing screening - a silent revolution. The New England journal of medicine 2006; 354: 2151 - 2164

62. Matschke RG, Plath P. Zur Friiherkennung von Horstorungen. Eine einfache Methode der Reihenuntersuchung bei Neugeborenen. HNO 1985; 33: 40-44

63. Norris VW, Amos KS, Hanks WD, Xia X, Nance WE, Pandya A. Does universal newborn hearing screening identify all children with GJB2 (Connexin 26) deafness? Penetrance of GJB2 deafness. Ear and hearing 2006; $27: 732-741$

64. Schade G, Kothe C, Ruge G, Hess M, Meyer CG. Screening auf Connexin 26-Mutationen mit Wangenschleimhautmaterial zur nichtinvasiven Diagnostik genetisch bedingter Innenohrschwerhorigkeit. LaryngoRhino Otologic 2003; 82; 397-401

65. Schade G, Bolz SS, Bolz H. Wangenabstriche zur genetischen Abklarung kindlicher Innenohrschwerhorigkeit. Online-Publikation beim GMS (German Medical Science)-Verlag, 2004

66. Bolz H, Schade G, Ehtner S, Kothe C, Hess M, Gal A. Phenotypic variability of non-syndromic hearing loss in patients heterozygous for both c. $35 \mathrm{delG}$ of GJB2 and the $342-\mathrm{kb}$ deletion involving GJB6. Hearing Research 2004; 188: 42-46

67. Bolz H, Bolz S, Schade G, Kothe C, Mohrmann G, Pohl U, Hess M, Gal A. Impaired calmodulin binding of myosin $7 \mathrm{~A}$ causes autosomal dominant hearing loss (DFNA 11). Human Mutation 2004; 24: 274-275

68. Schrijver I, Gardner P. Hereditary sensorineural hearing loss: advan $\neg$ ces in molecular genetics and mutation analysis. Expert review of molecular diagnostics 2006; 6: 375-386

69. Birkenhiiger R, Aschendorff A, SchipperJ, LaszigR. Nicht-syndromalehereditare Schwerhorigkeiten. Laryngo Rhino Otol 2007; 86: 299-309

70. Gross M. Universelles Horscreening bei Neugeborenen - Empfehlungen zu Organisation und Durchfiihrung des universellen Neugeborenen-Screenings auf angeborene Horstorungen in Deutschland. Laryngo-Rhino-Otol 2005; 84: 801-808

71. Gross M, Buser K, Freitag U, Hess MM, Hesse V, Hildmann A, Hildmann H, Hippel K, Lenarz T, Lindbauer-Eisenach Ц Plinkert P, Pohlandt F, Ptok M, Reuter G, Rossi R, Schnitzer S, Thyen U, Vetter K. Universelles Horsereening bei Neugeborenen - Empfehlungen zu Organisation und Durchfiihrung des universellen NeugeborenenScreenings auf angeborene Horstorungen in Deutschland. Zeitschrift fur Geburtshilfe und Neonatologie 2004; 208: 239-245

72. Nennstiel-Ratzel U, Arenz S, Wildner M, Kries RV, Liebl B. Neue Heraus-forderungen fur das Screening-Zentrum im Bayerischen Landesamt fur Gesundheit und Lebensmittelsicherheit. Gesundheitswesen 2004; 66: 8-12

73. Delb W, Merkel D, Pilorget K, SchmittI, Plinkert PK. Effectiveness of a TEOAE-based screening program. Can a patient-tracking system effectively be organized using modern information technology and central data management. European archives of Oto-Rhino-Laryngol 2004; 261: 191-196

74. Finckh-Kramer U, Spormann-Lagodzinski ME, Nubel K, Hess M, Gross M. Wird die Diagnose bei persistierenden kindlichen Horstorungen immer noch zu spat gestellt? HNO 1998; 46: 598-602

75. Canale A, Favero E, Lacilla M, Recchia E, Schindler A, Roggero N, Albera R. Age at diagnosis of deaf babies: a retrospective analysis high lighting the advantage of newborn hearing screening. International journal of pediatric otorhinolaryngology 2006; 70: 1283-1289 
76. Shehata-Dieler WE, Dieler R, Keim R, Finkenzeller P, Dietl J, Helms J. Universelle Horscreening-Untersuchungen bei Neugeborenen mit dem BERAphon. Laryngo Rhino Otol 2000; 79: 69-76

77. Stuhrmann NC, Limberger A, Schade G. Management bei kindlicher Schwerhorigkeit - Teil I: Atiologie. HNO 2008; im Druck

78. Schmuziger N, LodwigA, Probst R. Influence of artifacts and pass/refer criteria on otoacoustic emission hearing screening. International journal of audiology 2006; 45: 67-73

79. Schmidt CM. Verfugbare Geratetechnologien. Vortrag in Munster beim Symposion ,Horscreening in NRW", am 5.9.2007

80. Foerst A, Beutner D, Lang-Roth R, Huttenbrink KB, von Wedel H, Walger M. Prevalence of auditory neuropathy/synaptopathy in a population of children with profound hearing loss. International journal of pediatric otorhinolaryngology 2006; 70:1415-1422

81. Ngo RV, Tan HK, Balakrishnan A, Lim SB, Lazaroo DT. Auditory neuropathy/auditory dys-synchrony detected by universal newborn hearing screening. International journal of pediatric otorhinolaryngology 2006; 70: 1299-1306

82. Paradise JL, Campbell TF, Dollaghan CA, Feldman HM, Bernard BS, Colborn DK, Rockette HE, Janosky JE, Pitcairn DL, Kurs-Lasky M, Sabo DL, Smith CG. Developmental outcomes after early or delayed insertion of tympanostomy tubes. The New England journal of medicine 2005: $353: 576-586$

83. Paradise JL, Feldman HM, Campbell TF, Dollaghan CA, Colborn DK, Bernard BS, Rockette HE, Janosky JE, Pitcaim DL, Sabo DL, Kurs-Lasky M, Smith CG. Effect of early or delayed insertion of tympanostomy tubes for persistent otitis media on developmental outcomes at the age of three years. The New England journal of medicine 2001; 344:1179-1187

84. Coninx IF. Audiopadagogik. Vortrag auf der Fortbildungsveranstaltung "Hor- und Sprachdiagnostik im Vorschulalter" Bonn: 10. 10. 2007

85. Wiesner T, Bohnert A, Massinger C. Konsenspapier der DGPP zur Horgerate-Versorgung bei Kindern. Vers. 3. 0.; 2007; verabschiedet DGPP-Jahrestagung 2002 - Erlangen, revidiert und erweitert 2006 Heidelberg und 2007 Innsbruck; 1-12

86. Kiese-Himmel C, Reeh M. Orale Sprachentwicklung bilateral schallempfindungsgestorter Kinder - ein empirischer Langsschnitt. Gesundheitswesen 2007; 69: 249-255

87. Grosse SD, Ross DS. Cost savings from universal newborn hearing screening. Pediatrics 2006; 118: 844-845

88. Kunze S, Schnell-Inderst P, Hessel F, Grill E, Nickisch A, Siebert U, von Voss H, Wasem J. Horscreening fur Neugeborene - ein Health Technology Assessment der medizinischen Effektivitat und der okonomischen Effizienz. Schriftenreihe HTA des DIMDI Dauben HP, Riither A, WArda F, (Hrsg): Niebull: Medicombooks. de, 2004; 12: ISBN 389906-735-5, http://www.dimdi.de/HTA

89. Cao-Nguyen MH, Kos MI, GuyotJP. Benefits and costs of universal hearing screening programme. International journal of pediatric otorhinolaryngology 2007; 71: 1591 -1595

90. Fleer A. Erfassung aller horgeschadigten Neugeborenen: OAE-Screening bedarf der Finanzierung. zu dem Beitrag von Prof. Dr. med. Peter Karl Plinkert Dr. med. Wolfgang Delb, Priv. -Doz. Dr. med. Stefan Graber in Heft 3/2002. Deutsches Arzteblatt 2002; 99: 1442

91. BuserK, Bietendiiwel A, Krauth CJalilvandN, MeyerS, ReuterG, Stolle S, Altenhofen L, Lenarz T. Modellprojekt Neugeborenen-Horscreening in Hannover (Zwischenergebnisse). Gesundheitswesen 2003; 65:200203

92. Schonweiler R, Tioutou E, Tollgczko R, Pankau R, Ptok M. Horscreening mit automatisch bewertetenTESAE und einem neuen Verfahren automatisch bewerteter FAEP. Optimierung und Feldversuch. HNO 2002: 50: 649-656

93. Schonweiler R. Universelles Neugeborenen-Horscreening: Begriindung, Methodik und gegenwartiger Stand. Vortrag in Munster beim Symposion „Horscreening in NRW", am 5. 9. 2007

94. Delb W. Erfassung aller horgeschadigten Neugeborenen: Schlusswort zu dem Beitrag von Prof. Dr. med. Peter Karl Plinkert, Dr. med. Wolfgang Delb, Priv.-Doz. Dr. med. Stefan Graber in Heft 3/2002. Deutsches Arzteblatt 2002: 99:1442

95. Delb W. Universelles Neugeborenenhorscreening in Deutschland. Wer ist "am Zug"? HNO 2002; 50: 607-610
96. Finitzo T, Brookhauser P, Epstein S, EhrenbergA et al. Joint Committee on Infant hearing Year 2000 Position Statement: Principles and Guidelines for Early Detection and Intervention Programs. Pediatrics 2000; 106:798-817

97. Wrightson AS. Universal newborn hearing screening. American family physician 2007; 75: 1349-1352

98. Babac S, Djeric D, Ivankovic Z. Newborn hearing screening. Srpski arhiv zacelokupno lekarstvo2007; 135: 264-268

99. Dedau F, Doyen A, Robillard T, de Varebeke SJ. Comparison of hearing screening programs between one step with transient evoked otoacoustic emissions (TEOAE) and two steps with TEOAE and automated auditory brainstem response. The Laryngoscope 2005; 115:1957-1962

100. Helge T, Werle E, Barnick M, Wegner C, Ruhe B, Aust G, Rossi R. Sequen-zielles Neugeborenen-Horscreening (TEOAE/AABR) reduziert Recall-Rate. Erfahrungen in einem Berliner Perinatalzentrum. HNO 2005; $53: 655-60$

101. Finckh-Kramer U, Cross M, Bartsch M, Kewitz G, Versmold H, Hess M. Horscreening von Neugeborenen mit Risikofaktoren. HNO 2000; 48: $215-220$

102. Meyer C, Witte], Hildmann A, Hetmecke KH, Schunck KU, Maul K, Franke U, Fahnenstich H, Rabe H, Rossi R, Hartmann S, Gartner L Neonatal screening for hearing disorders in infants at risk: incidence, risk factors, and follow-up. Pediatrics 1999; 104: 900-904

103. Sitka U, Rasinski C, Gall V. Ergebnisse eines NeugeborenenHorscreenings mittels transitorisch evozierter otoakustischer Emissionen. Zeitschrift fur Geburtshilfe und Neonatologie 1995; 199: 71-77

104. Renter G, Bbrdgen F, Dressier F, Schafer S, Hemmanouil I, Schonweiler R, Lenarz T. Neugeborenenhorscreening mit dem automatisierten Messgerat Echosensor fur otoakustische Emissionen. Eine vergleichende Untersuchung. HNO 1998; 46: 932-941

105. BretschneiderJ, Maier H, Hess M. Leuwer R. Aufwand und Ergebnisse eines universellen ERANeugeborenenhorscreenings mit dem ALGO® portable. Laryngo Rhino Otol 2001; 80: 357-364

106. Heinemann M, Bohnert A. Horscreening bei Neugeborenen. Vergleichende Untersuchungen und Kostenanalysen mit verschiedenen Geraten. Laryngo Rhino Otol 2000; 79: 453-458

107. Massinger C, Lippert KL, Keilmann A. Verzogerung in der Horbahnreifung - Differentialdiagnose bei Horstorungen im Sauglingsalter. HNO 2004; 52: 927-934

108. Lesinski-SchiedatA, UlgA, WarneckeA, Heermann R, Bertram B, Lenarz T. Kochleaimplantation bei Kindern im 1. Lebensjahr: Vorlaufige Ergebnisse. HNO 2006; 54: 565-572

109. Kolski C, he Driant B, Lorenzo P, Vandromme L, Strunski V. Early hearing screening: what is the best strategy? International journal of pediatric otorhinolaryngology 2007; 71:1055-1060

110. Korres SG, Balatsouras DG, Nikolopoulos T, Korres GS, Ferekidis E Making universal newborn hearing screening a success. International pediatric otorhinolaryngology 2006; $70: 241-246$

111. Lang-Roth R, Walger M, Wedel H von, Neuschaefer-Rube C, Angestein W, Schade G. Projektbeschreibung: Implementierung eines qualitats-gesicherten, universellen Neugeborenen-Horscreenings (qUNHS) in NRW - Modellprojekt Nordrhein; Verbundprojekt Nordrhein mit den Universitatskliniken Aachen, Bonn, Diisseldorf und Koln 2007

112. Hess M, Neumann K, Nawka T, Gross M, Wiesner T. Vorlage des Konsensuspapiers der Arbeitsgemeinschaft Neugeborenen-Horscreening: Grundlagen zur Qualitatssicherung eines universellen NeugeborenenHorscreenings, empfohlen von der Deutschen Gesellschaft fur Phoniatric \& Padaudiologic (DGPP) 2007

113. Weichbold V, Welzel-Muller K. Universelles NeugeborenenHorscree-ning: Einstellungen und Angste der Mutter. HNO 2000; 48 606-612

114. Young A, Tattersall $\mathrm{H}$. Universal newborn hearing sereening and early identification of deafness: parents' responses to knowing early and their expectations of child communication development. Journal of deaf studies and deaf education 2007; 12: 209-220

115. Arnold CL, Davis TC, Humiston SG, Bocchini]AJr, Bass PF3rd, Bocchini A, Kennen EM, White K. Forsman I. Infant hearing screening: stakeholder recommendations for parent-centered communication. Pediatrics 2006; 117: 341-354 